\title{
Structural shape optimization using self-adjusted convex approximation
}

\author{
T.T. Chung and C.H. Chiou
}

\begin{abstract}
This study researches the applications of Self-Adjusted Convex Approximation (SACA) in structural shape optimization problems. The B-spline curve is adopted as the mathematical representation of the structural shapes. The SACA method is based on the CONvex LINearization (CONLIN) method and has better accuracy and convergent rate. Numerical examples are offered and the results show that the proposed method is effective in the structural shape design.
\end{abstract}

Key words convex approximation method, approximation concepts, shape optimization, structural optimization

1

\section{Introduction}

Structural optimum design is to select the design variables such that the weight of the structure is minimized while the constraints are satisfied. The numerical form is as follows:

$\operatorname{minimize} F(\mathbf{x})$,

subject to

$h_{q}(\mathbf{x}) \leq 0, \quad q=1,2, \ldots, n_{C}$,

where $\mathbf{x}$ denotes the design vector, $x_{i}$ represents the $i$-th component of the design vector, $F(\mathbf{x})$ is the objective

Received April 2, 1999

Revised manuscript received August 3, 1999

T.T. Chung and C.H. Chiou

Department of Mechanical Engineering, National Taiwan University, Taipei, 10617, Taiwan

e-mail: ttchung@ccms.ntu.edu.tw function, $h_{q}(\mathbf{x})$ denotes the $q$-th constraint and $n_{C}$ represents the number of constraints.

Since structural optimization problems are characterized by computationally expensive function evaluations, many approaches have been proposed in the recent decade to resolve this problem. Amongst these, the most widely used approach applies approximation concepts to generate a sequence of separable subproblems that can be solved iteratively. The most commonly used approximation method is based on the use of the first-order Taylor series expansion in terms of the linear and the reciprocal design variables. Fleury and Braibant (1986) presented a hybrid form of linear and reciprocal approximation, called convex linearization (CONLIN), which is more conservative. For most cases, CONLIN is useful in structural optimization due to its conservativeness but it is still inaccurate for some design problems. Svanberg (1987) proposed the method of moving asymptotes (MMA) that is also based on a special type of convex approximation. Kirsch (1995) developed a scaling factor for a stiffness matrix and approximated the displacements, stresses and forces.

Fadel et al. (1990) developed a two-point approximation, which is a linear approximation in terms of intermediate variables $x_{i}^{p_{i}}$. The exponents $p_{i}(i=1,2, \ldots, n)$ are determined by matching function derivatives at the previous data point. Wang and Grandhi (1995) presented the improved two-point function approximations, which not only match the gradients at the previous point, but also match the function value at the previous point. However, this method is limited largely by its difficulty in obtaining the exponents $p_{i}$. The solution even does not exist due to serious nonlinearity of the exponential approximate function. In a related work, Wang et al. (1996) developed a multipoint approximation, which used Hermite interpolation concepts and the multipoint information generated at the iterations of optimization. Salajegheh (1997) developed a three-point approximation which is expanded at the middle point and the value of the functions and the derivatives of the first and third points. Zhang and Fleury (1997) modified convex approximation 
for structural optimization. He applied a two-point fitting scheme to adjust the convexity of constraint approximations during the design process.

\section{2}

\section{Background}

\section{1}

\section{Shape representation}

In the first few papers concerning shape optimum design, the nodal coordinates of a finite element model were chosen as the design variables. The disadvantages of this were the large number of design variables and the difficulty of maintaining an adequate finite element mesh during the optimization iteration. Braibant and Fleury (1984) utilized the B-spline curve as the shape representation. The formulation of the B-spline curve can be expressed as

$$
\begin{aligned}
& P(u)=\sum_{i=1}^{n_{b}} P_{i} N_{n, k}(u), \quad 0 \leq u \leq u_{\max }, \\
& N_{i, 1}(u)= \begin{cases}1 & t_{i} \leq u \leq t_{i+1}, \\
0 & \text { otherwise }\end{cases} \\
& N_{i, k}(u)=\frac{\left(u-t_{i}\right) N_{i, k-1}(u)}{t_{i+k-1}-t_{i}}+\frac{\left(t_{i+k}-u\right) N_{i+1, k-1}(u)}{t_{i+k}-t_{i+1}},
\end{aligned}
$$

where $n_{b}$ is the number of the control nodes minus 1 , and $k$ is the degree of the B-spline curve.

The coordinates of the control nodes of the B-spline curves are chosen as the design variables in this study. The advantages of using the B-spline curve are its flexibility and reasonable geometric structural shape.

\section{2}

\section{Function approximations}

The simplest form is the linear approximation based on Taylor series, and it is shown as follows:

$h_{d}(\mathbf{x})=h\left(\mathbf{x}_{0}\right)+\left.\sum_{i=1}^{n} \frac{\partial h}{\partial x_{i}}\right|_{\mathbf{x}_{0}}\left(x_{i}-x_{i 0}\right)$

where $h_{d}(\mathbf{x})$ denotes the direct linear approximation function of $h(\mathbf{x})$ and $\partial h /\left.\partial x_{i}\right|_{\mathbf{x}_{0}}$ represent the sensitivities of $h(\mathbf{x})$ with respect to the $i$-th design variable at the original design $\mathbf{x}_{0}$.

In truss design problems, the cross-sectional area of the bar structure is often chosen as the design variable. It is advantageous to use reciprocals of the design variables to formulate the approximation. The reciprocal approximation can be expressed as $h_{r}(\mathbf{x})=h\left(\mathbf{x}_{0}\right)+\left.\sum_{i=1}^{n} \frac{\partial h}{\partial x_{i}}\right|_{\mathbf{x}_{0}}\left(x_{i}-x_{i 0}\right) \frac{x_{i 0}}{x_{i}}$,

where $h_{r}(\mathbf{x})$ is the reciprocal approximation of $h(\mathbf{x})$.

Another approximation, called the conservative approximation (Starnes and Haftka 1979), generally referred to as CONLIN (Fleury and Braibant 1986), is a hybrid form of linear and reciprocal approximation. The selection of variables is made based on the signs of the first partial derivatives, that is, a direct variable for a positive derivative and a reciprocal value for a negative one. It can be written in the following form:

$h_{c}(\mathbf{x})=h\left(\mathbf{x}_{0}\right)+\left.\sum_{i}^{(+)} \frac{\partial h}{\partial x_{i}}\right|_{\mathbf{x}_{0}}\left(x_{i}-x_{i 0}\right)+$

$\left.\sum_{i}^{(-)} \frac{\partial h}{\partial x_{i}}\right|_{\mathbf{x}_{0}}\left(x_{i}-x_{i 0}\right) \frac{x_{i 0}}{x_{i}}$,

where $h_{c}(\mathbf{x})$ denotes the convex approximation of $h(\mathbf{x})$ and $\sum_{i}^{(+)}\left(\sum_{i}^{(-)}\right)$represents summation over the variables in which sensitivities are positive (negative).

CONLIN has shown good convergent properties in dealing with structural optimization problems due to its conservativeness. However, although the convex approximation achieves stable numerical results, the optimum process may converge slowly if approximate functions are too conservative or still oscillate if they are not conservative enough.

Fadel et al. (1990) developed a two-point approximation, which is a linear approximation in the variables

$y_{i}=x_{i}^{p_{i}}$.

It can be expressed in the following form:

$h_{t}(\mathbf{x})=h\left(\mathbf{x}_{0}\right)+\left.\sum_{i=1}^{n} \frac{\partial h}{\partial x_{i}}\right|_{\mathbf{x}_{0}}\left[\left(\frac{x_{i}}{x_{i 0}}\right)^{p_{i}}-1\right] \frac{x_{i 0}}{p_{i}}$.

Then, $p_{i}$ can be determined to match the first derivatives at another design point. That is,

$p_{i}=1+\frac{\ln \left[\left(\frac{\partial h}{\partial x_{i}}\right)_{\mathbf{x}_{1}} /\left(\frac{\partial h}{\partial x_{i}}\right)_{\mathbf{x}_{0}}\right]}{\ln \left(x_{1 i} / x_{0 i}\right)}$.

Wang and Grandhi (1995) presented a two-point adaptive nonlinear approximation which can be expressed as

$h_{\text {tana }}(\mathbf{x})=h\left(\mathbf{x}_{0}\right)+\sum_{i=1}^{n}\left(\left.\frac{\partial h}{\partial x_{i}}\right|_{\mathbf{x}_{0}}\left[\left(\frac{x_{i}}{x_{i 0}}\right)^{p_{i}}-1\right] \frac{x_{i 0}}{p_{i}}+\right.$

$\left.\frac{1}{2} \varepsilon_{2}\left(x_{i}^{p_{i}}-x_{i 0}^{p_{i}}\right)^{2}\right)$.

Differentiating (9), $n$ equations are obtained by matching the derivatives at the previous point. Another equation 
is obtained by matching the function value at the previous point. The $n+1$ equations are solved by numerical iteration method in order to determine $p_{i}$ and $\varepsilon_{2}$.

Zhang and Fleury (1997) modified the convex approximation as follows:

$h_{m c}(\mathbf{x})=h_{c}(\mathbf{x})+\frac{1}{2} \alpha\left\|x_{i}-x_{i 0}\right\|^{2}$,

where $\alpha$ can be determined by matching the function value at the previous point. If $\alpha$ is negative, a small positive value will be imposed.

3

\section{Self-adjusted convex approximation}

\section{1}

\section{Higher-order convex approximations}

Select intermediate variable $y_{i}=x_{i}^{r_{i}}$ if the derivatives $\left(\partial h / \partial x_{i}\right)_{\mathbf{x}_{0}}>0$ and $y_{i}=1 / x_{i}^{r_{i}}$ if $\left(\partial h / \partial x_{i}\right)_{\mathbf{x}_{0}}<0$. Then, the higher-order convex approximation can be written in the following form:

$h_{h}(\mathbf{x})=h\left(\mathbf{x}_{0}\right)+\left.\sum_{i}^{(+)} \frac{1}{r_{i}} \frac{\partial h}{\partial x_{i}}\right|_{\mathbf{x}_{0}}\left[\left(\frac{x_{i}}{x_{i 0}}\right)^{r_{i}-1} x_{i}-x_{i 0}\right]$

$\left.\sum_{i}^{(-)} \frac{1}{r_{i}} \frac{\partial h}{\partial x_{i}}\right|_{\mathbf{x}_{0}}\left[\left(\frac{x_{i}}{x_{i 0}}\right)^{r_{i}-1} x_{i}-x_{i 0}\right]\left(\frac{x_{i 0}}{x_{i}}\right)^{r_{i}}$,

$r_{i} \in R$,

where $r_{i}$ represents the order or degree of convexity of the constraint $h(\mathbf{x})$ with respect to the design variable $x_{i}$, which can be different for each design variable of each constraint at each iteration.

The convex approximation method can be considered as a special case of higher-order convex approximation of order 1 . The attractive property of this approximation is that higher order leads to more conservative results. Restated,

$h_{r_{i}}(\mathbf{x}) \geq h_{s_{i}}(\mathbf{x}), \quad$ if $r_{i} \geq s_{i}, \quad i=1,2, \ldots, n$.

The proof is shown in Appendix A.

\section{2}

\section{Self-adjusted convex approximations}

This method is based on higher-order convex approximation. The order $r_{i}$ for each design variable is automatically adjusted to prevent the underestimation or overestimation of the actual constraint values between two consecutive iteration steps. For a lower value, the approximate constraint values probably are underestimated. Restated, the approximate constraint values are less conservative than the actual constraint values. Under such a condition, the design variable values of the solution of the approximate problem oscillate between consecutive iteration steps. This requires increasing the order to resolve this situation.

In this study, the orders $r_{i}$ for the design variable $x_{i}$ are simplified to be the same, i.e.

$r_{i}=r, \quad i=1,2, \ldots, n$.

This simplified form of SACA can be written as

$h_{a}(\mathbf{x})=h\left(\mathbf{x}_{0}\right)+\left.\sum_{i}^{(+)} \frac{1}{r} \frac{\partial h}{\partial x_{i}}\right|_{\mathbf{x}_{0}}\left[\left(\frac{x_{i}}{x_{i 0}}\right)^{r-1} x_{i}-x_{i 0}\right]$

$\left.\sum_{i}^{(-)} \frac{1}{r} \frac{\partial h}{\partial x_{i}}\right|_{\mathbf{x}_{0}}\left[\left(\frac{x_{i}}{x_{i 0}}\right)^{r-1} x_{i}-x_{i 0}\right]\left(\frac{x_{i 0}}{x_{i}}\right)^{r}$,

$r \in R$,

In the first iteration, the convex or higher-order convex approximation can be used as the approximation method. After the first iteration, the optimum design vector $\mathbf{x}_{1}$, the actual constraint value $h\left(\mathbf{x}_{0}\right)$ and the approximate constraint function $h_{a}\left(\mathbf{x}_{1}\right)$ expanded at the original design point $\mathbf{x}_{0}$ can be obtained.

In the second iteration, the actual constraint value $h\left(\mathbf{x}_{1}\right)$ can be obtained by using finite element analysis. Therefore, $h\left(\mathbf{x}_{1}\right)$ can be easily compared with the estimated constraint value $h_{a}\left(\mathbf{x}_{1}\right)$. This is a situation in which $h_{a}\left(\mathbf{x}_{1}\right)<h\left(\mathbf{x}_{1}\right)$ implies that the approximation function of the first iteration is not conservative enough. Restated, it underestimates the actual constraint. Therefore, the order must be increased to avoid oscillation or even an infeasible design. In contrast, a situation in which $h_{a}\left(\mathbf{x}_{1}\right)>h\left(\mathbf{x}_{1}\right)$ implies that the degree of convexity is conservative enough. However, it is probably too large and will ultimately result in a slower convergence. Therefore, the order should be decreased to accelerate the convergence.

The criterion for adjusting the order is that the approximation function does not underestimate or overestimate the actual constraint value at $\mathbf{x}_{1}$. That is, $h_{a}\left(\mathbf{x}_{1}, r\right)$ $=h\left(\mathbf{x}_{1}\right)$. Substituting it into (14) yields

$h\left(\mathbf{x}_{0}\right)+\left.\sum_{i}^{(+)} \frac{1}{r} \frac{\partial h}{\partial x_{i}}\right|_{\mathbf{x}_{0}}\left[\left(\frac{x_{i 1}}{x_{i 0}}\right)^{r-1} x_{i 1}-x_{i 0}\right]+$

$\left.\sum_{i}^{(-)} \frac{1}{r} \frac{\partial h}{\partial x_{i}}\right|_{\mathbf{x}_{0}}\left[\left(\frac{x_{i 1}}{x_{i 0}}\right)^{r-1} x_{i 1}-x_{i 0}\right]\left(\frac{x_{i 0}}{x_{i 1}}\right)^{r}=$

$h\left(\mathbf{x}_{1}\right)$.

From the above equation, the proper order $r$ of the constraint can be determined. The computed order is adopted as the order of the higher-order convex approximation in the optimization process.

This method has two distinct advantages over the twopoint approximation methods. First, (15) always contains 
a solution because the larger $r$ leads to more conservative (larger) approximation function. So, the order $r$ in (15) can be easily determined. Restated, SACA does not need to obtain this order by using the least square method or even by performing another optimization algorithm. Second, the order of SACA has some kind of physical meaning, it can be roughly estimated even without performing the two-point scheme. It can be less than 1 and even be negative, in other words, it is less conservative than CONLIN.

4

\section{Numerical examples}

\section{1}

\section{Cantilever beam with stress constraint}

This example consists of finding the minimum weight of the cantilever beam shown in Fig. 1. The beam is loaded with a concentrated load $P=150 \mathrm{lb}$ at the tip and has material properties of modulus of elasticity $E=$ $10.3 \times 10^{6} \mathrm{psi}, \nu=0.3$ and $\varrho=0.1 \mathrm{lb} / \mathrm{in}^{3}$. The thickness of the beam is $b=1.0 \mathrm{inch}$ and initial height is 30 inches. The stress constraints $\sigma_{\text {admi }}$ on the elements are 500 psi.

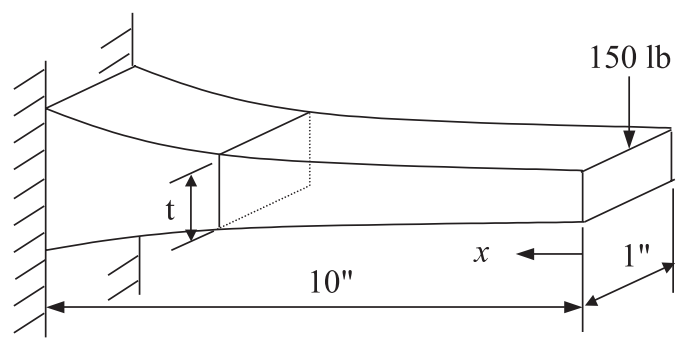

Fig. 1 Cantilever beam with stress constraint

A fifth degree B-spline curve with seven control nodes is utilized as the shape representation, and the vertical coordinates of the seven control nodes are chosen as the design variables. The analytical solution of the optimum shape to this problem can be obtained in the following form:

$t=\sqrt{\frac{6 P x}{b \sigma_{\mathrm{admi}}}}$.

In Fig. 2, the computed optimum shape of this example is compared with the theoretical optimum shape. The iteration histories of CONLIN and SACA are shown in Fig. 3. Table 1 lists the orders for SACA in the optimum iterations. This table indicates that most orders are less than 1 . This finding implies that CONLIN is too conservative for this problem. Consequently, CONLIN requires three more iterations than SACA to converge.

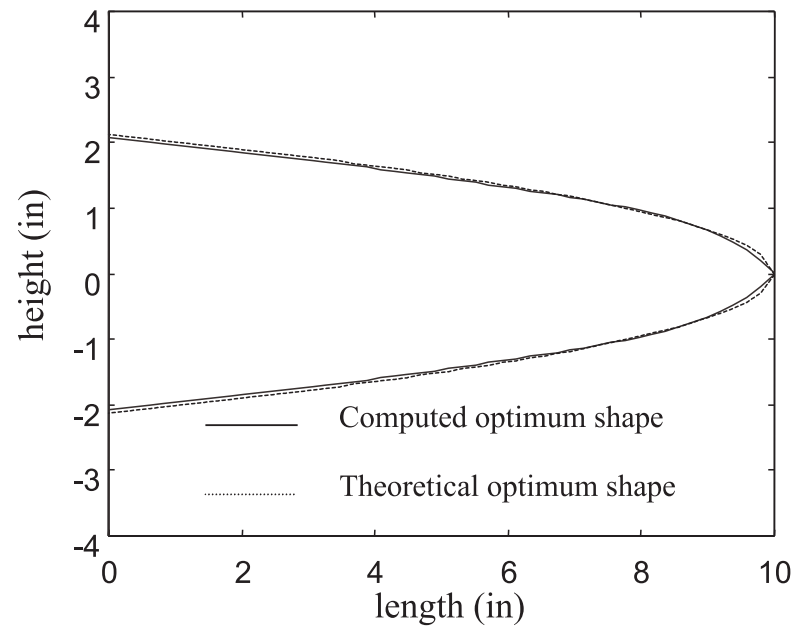

Fig. 2 Optimum shapes of example 4.1

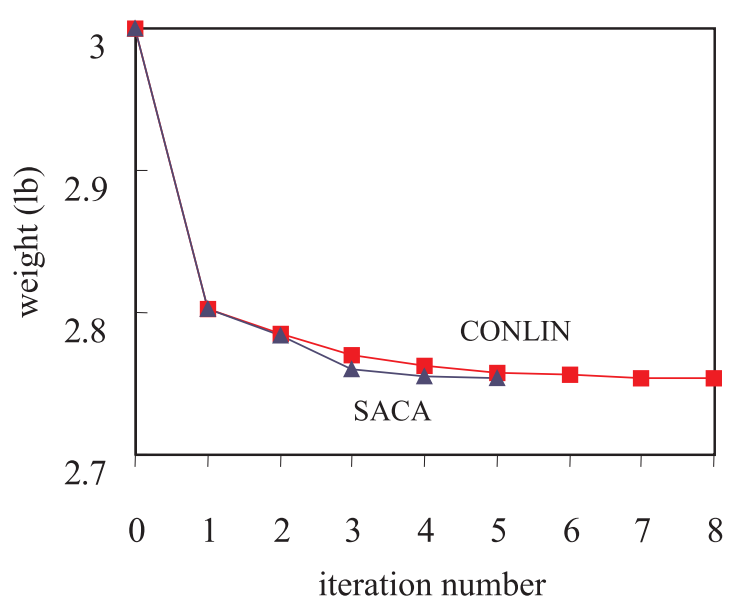

Fig. 3 Iteration history of example 4.1

Table 1 The orders for SACA of example 4.1

\begin{tabular}{|c|c|c|c|c|c|}
\hline Iteration no. & 1 & 2 & 3 & 4 & 5 \\
\hline Constraint & 1.000 & 1.274 & -0.358 & -0.491 & -0.884 \\
\hline Constraint & 1.000 & 1.785 & 1.598 & 0.430 & 1.133 \\
\hline Constraint & 1.000 & 1.796 & 0.828 & 0.078 & 0.781 \\
\hline Constraint & 1.000 & 1.580 & -0.504 & -0.142 & -0.801 \\
\hline Constraint 5 & 1.000 & 0.467 & -1.207 & 0.188 & 0.737 \\
\hline Constraint 6 & 1.000 & -0.946 & 0.386 & -0.158 & -0.878 \\
\hline Constraint 7 & 1.000 & -0.787 & 0.662 & 0.166 & 0.655 \\
\hline Constraint & 1.000 & 0.342 & 0.894 & -0.352 & -0.815 \\
\hline Constraint 9 & 1.000 & 0.556 & -0.251 & 0.482 & 0.588 \\
\hline Constraint 10 & 1.000 & 0.959 & -0.866 & -0.867 & -0.921 \\
\hline Constraint 11 & 1.000 & 1.386 & -0.571 & -0.546 & -0.883 \\
\hline
\end{tabular}

\section{2}

\section{Cantilever beam with frequency constraint}

A cantilever beam with concentrated mass $38.64 \mathrm{lb}$ at the tip is shown in Fig. 4. It has material properties of 


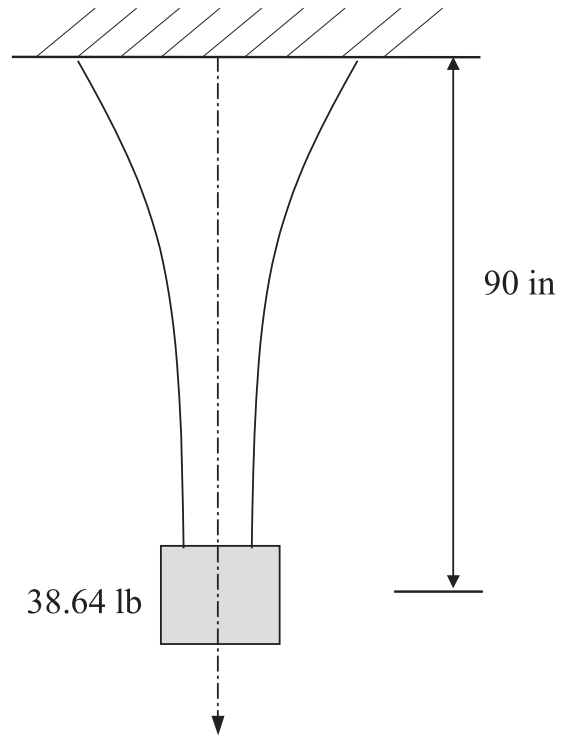

Fig. 4 Cantilever beam with frequency constraint

modulus of elasticity $E=10.3 \times 10^{6} \mathrm{psi}, \nu=0.3$ and material density $\varrho=0.1 \mathrm{lb} / \mathrm{in}^{3}$. The initial cross-sectional area is $9.2 \mathrm{in}^{2}$ and the first axial natural frequency is constrained to be greater than $410 \mathrm{~Hz}$.

Figure 5 shows the optimization processes of this example and iteration histories of the weight for SACA and CONLIN. According to Fig. 5, some iteration results of CONLIN fall into the infeasible region, where the first natural frequency violates the design constraint. This observation implies that CONLIN is not conservative enough for this example. To illustrate the underestimation of the constraint using convex approximation, the natural frequency in the iteration history is also shown in Fig. 6. Table 2 lists the orders of SACA at each iteration step. Table 2 reveals that most orders are greater than 1 . Restated, CONLIN is not conservative enough. The optimal and theoretical cross-sectional area for the cantilever beam is shown in Fig. 7.

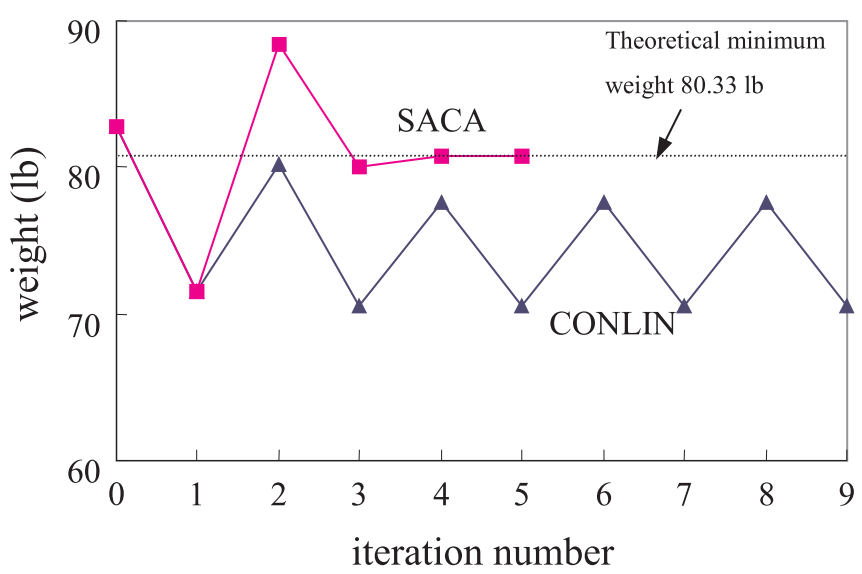

Fig. 5 Iteration history of example 4.2

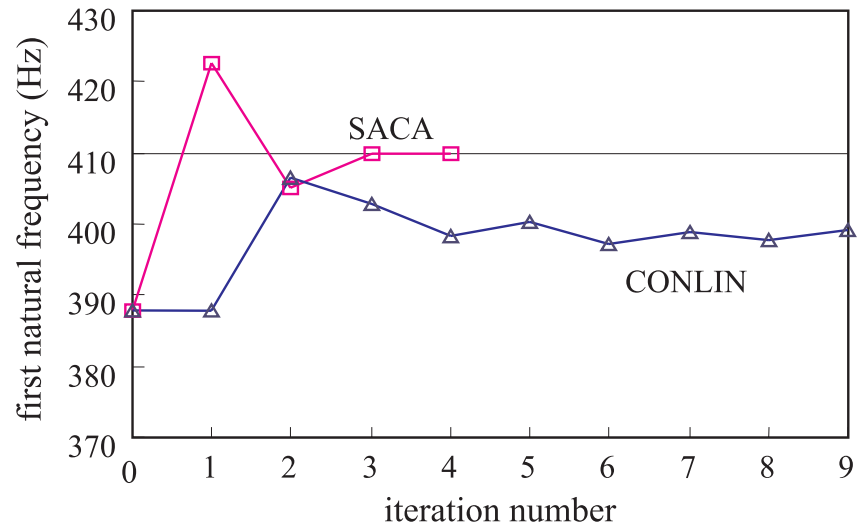

Fig. 6 Natural frequency in the optimization process

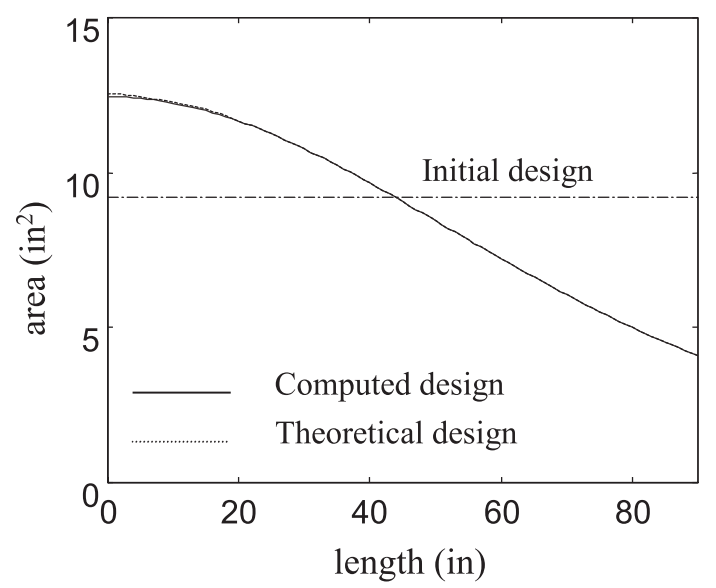

Fig. 7 Optimal and theoretical cross-sectional area $\left(\mathrm{in}^{2}\right)$ for example 4.2

Table 2 The orders for SACA of example 4.2

\begin{tabular}{llllll}
\hline Iteration no. & 1 & 2 & 3 & 4 & 5 \\
\hline \hline order & 1.00 & 3.90 & 0.32 & 2.44 & 2.61 \\
\hline
\end{tabular}

\section{3}

Torque arm

The structure shown in Fig. 8 is loaded simultaneously by a traction and mainly a bending force in the right side. Fixations are imposed along the circular hole in the left side. It has material properties of modulus of elasticity $E=2.074 \times 10^{7} \mathrm{~N} / \mathrm{cm}^{2}, \nu=0.3$ and material density $\varrho=7.81 \times 10^{-3} \mathrm{~kg} / \mathrm{cm}^{3}$. The thickness of the torque arm is $0.3 \mathrm{~cm}$ and the stress constraints on the elements are $40 \mathrm{kN} / \mathrm{cm}^{2}$.

A fourth degree B-spline curve with seven control nodes is adopted as the shape representation of the outside contour of the torque arm and another cubic B-spline curve with five control nodes as the representation of the inside contour. Figure 9 compares CONLIN and SACA 


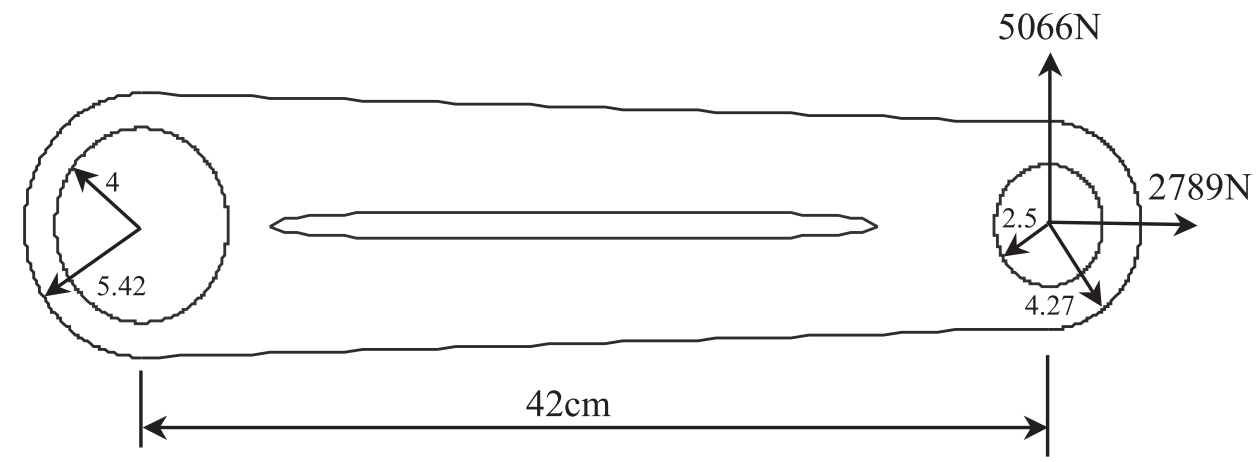

Fig. 8 Initial shape of torque arm

with respect to the iteration history of the structural weight. The optimum shape of this example is shown in Fig. 10. The optimization history of torque arm shape is shown in Fig. 11. In this example, the CONLIN method is too conservative and takes four more iterations to converge than SACA.

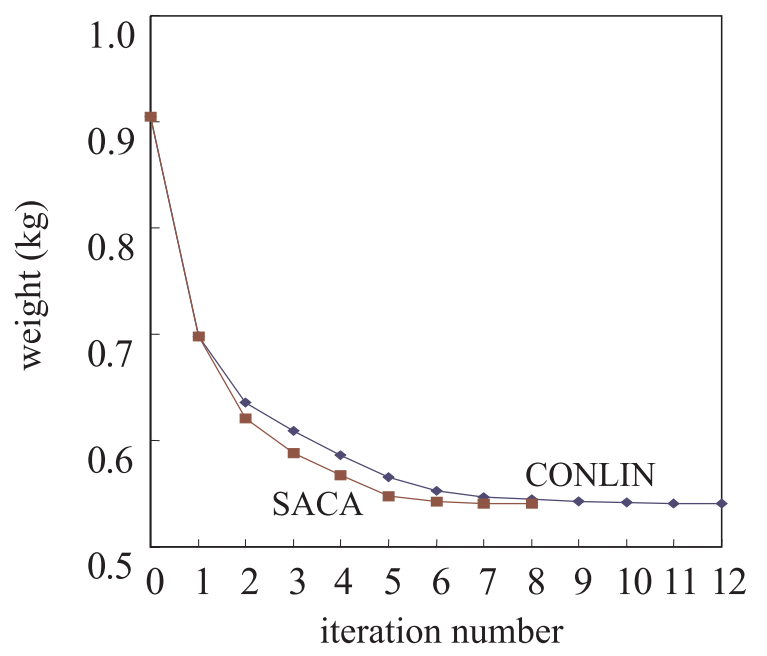

Fig. 9 Iteration history of example 4.3

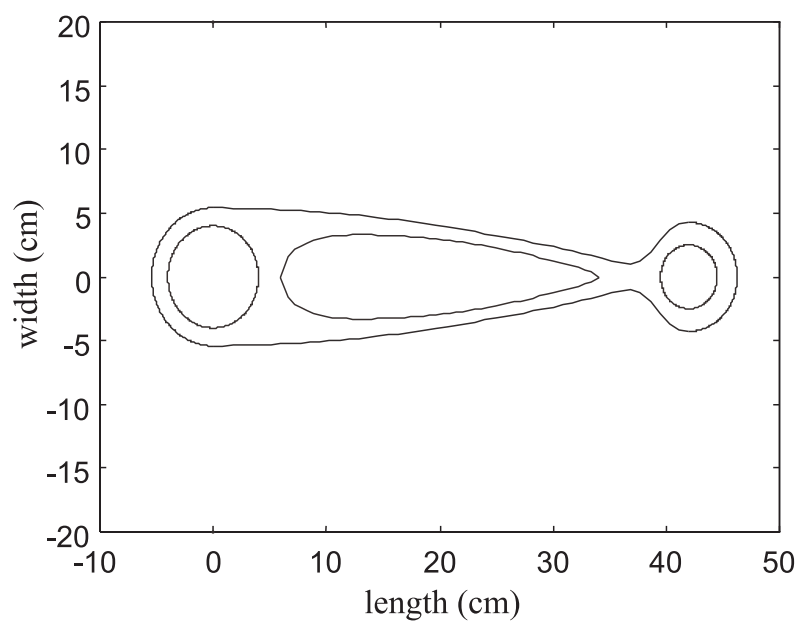

Fig. 10 Optimum shape of torque arm

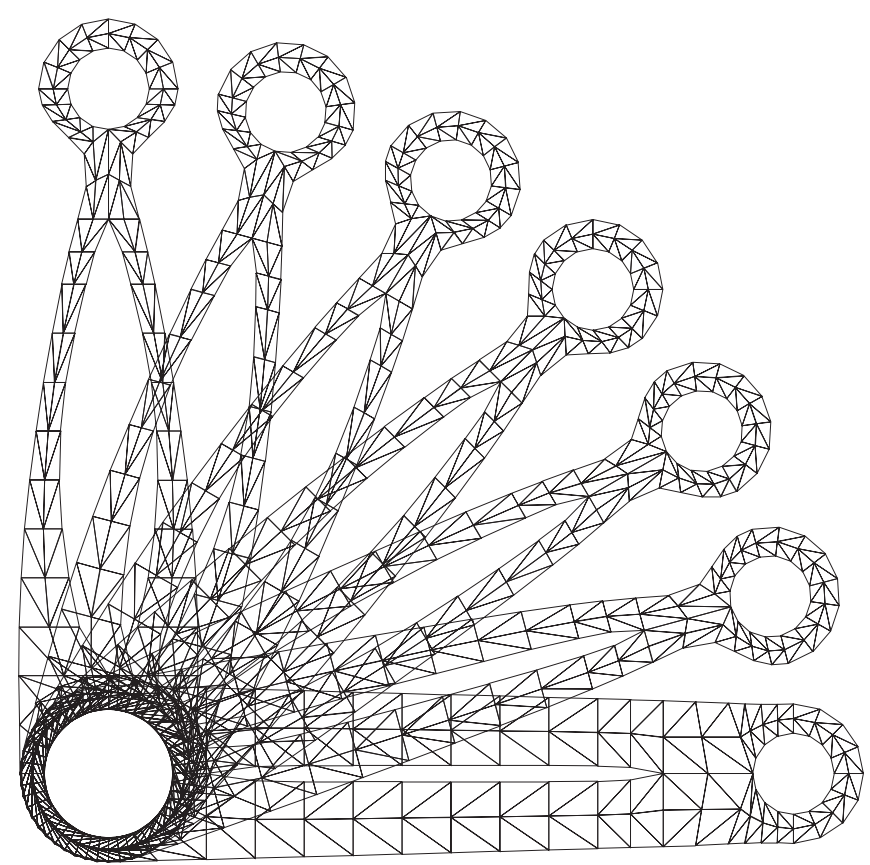

Fig. 11 Optimization history of the torque arm shape

5

\section{Conclusions}

This study utilizes the self-adjusted convex approximation (SACA) method in the applications of structural optimization. The SACA method can be considered as a general form of convex approximation. It can adjust the degree of convexity of the approximate constraint function to prevent underestimation or overestimation of the actual constraint. The method has an attractive property in that it yields higher constraint values with a higher order. Consequently, it is easy to adjust the proper order in the next design iteration. Out of the three examples, some approximation methods are too conservative and result in a slowly convergent rate; some are not conservative enough and result in an oscillating iteration history and even an infeasible design. The SACA method can adjust the order 
to avoid these situations and obtain quite satisfactory results.

\section{References}

Braibant, V.; Fleury, C. 1984: Shape optimal design using B-spline. Comp. Meth. Appl. Mech. Engng. 44, 247-267

Fadel, G.M.; Riley, M.F.; Barthelemy, J.M. 1990: Two point exponential approximation method for structural optimization. Struct. Optim. 2, 117-124

Fleury, C.; Braibant, V. 1986: Structural optimization: a new dual method using mixed variable. Int. J. Num. Meth. Engng. 23, 409-428

Kirsch, U. 1995: Improved stiffness-based first order approximations for structural optimization. AIAA J. 33, 143-150

Salajegheh, E. 1997: Optimum design of plate structures using three-point approximation. Struct. Optim. 13, 142-147

Starnes, J.H., Jr.; Haftka, R.T. 1979: Preliminary design of composite wings for buckling, stress and displacement constraints. J. Aircraft 16, 564-570

Svanberg, K. 1987: The method of moving asymptotes - a new method for structural optimization. Int. J. Num. Meth. Engng. 24, 359-373

Wang, L.P.; Grandhi, R.V. 1995: Improved two-point function approximations for design optimization. AIAA J. 33, $1720-1727$

Wang, L.P.; Grandhi, R.V.; Canfield, R.A. 1996: Multivariate Hermite approximation for design optimization. Int. J. Num. Meth. Engng. 39, 787-803

Zhang, W.H.; Fleury, C. 1997: A modification of convex approximation methods for structural optimization. Comp. \& Struct. 64, 89-95

\section{Appendix A: Conservative tendency of higher-order convex approximation}

To prove the conservative tendency of the higher-order convex approximation method, two different real numbers $r_{i}$ and $s_{i}$ are chosen as the degree convexity of (8) and $r_{i} \geq s_{i}$.

Then, $h_{r_{i}}(\mathbf{x}), h_{s_{i}}(\mathbf{x})$ can be expressed as

$$
\begin{aligned}
& h_{r_{i}}(\mathbf{x})=h\left(\mathbf{x}_{0}\right)+\left.\sum_{i}^{(+)} \frac{1}{r_{i}} \frac{\partial h}{\partial x_{i}}\right|_{\mathbf{x}_{0}}\left[\left(\frac{x_{i}}{x_{i 0}}\right)^{r_{i}-1} x_{i}-x_{i 0}\right]+ \\
& \left.\sum_{i}^{(-)} \frac{1}{r_{i}} \frac{\partial h}{\partial x_{i}}\right|_{\mathbf{x}_{0}}\left[\left(\frac{x_{i}}{x_{i 0}}\right)^{r_{i}-1} x_{i}-x_{i 0}\right]\left(\frac{x_{i 0}}{x_{i}}\right)^{r_{i}},
\end{aligned}
$$

$$
\begin{aligned}
& h_{s_{i}}(\mathbf{x})=h\left(\mathbf{x}_{0}\right)+\left.\sum_{i}^{(+)} \frac{1}{s_{i}} \frac{\partial h}{\partial x_{i}}\right|_{\mathbf{x}_{0}}\left[\left(\frac{x_{i}}{x_{i 0}}\right)^{s_{i}-1} x_{i}-x_{i 0}\right]+ \\
& \left.\sum_{i}^{(-)} \frac{1}{s_{i}} \frac{\partial h}{\partial x_{i}}\right|_{\mathbf{x}_{0}}\left[\left(\frac{x_{i}}{x_{i 0}}\right)^{s_{i}-1} x_{i}-x_{i 0}\right]\left(\frac{x_{i} 0}{x_{i}}\right)^{s_{i}} .
\end{aligned}
$$

Subtracting (18) from (17) gives

$h_{r_{i}}(\mathbf{x})-h_{s_{i}}(\mathbf{x})=\sum_{i}^{(+)} \alpha_{i} \kappa\left(\xi_{i}, r_{i}, s_{i}\right)+$

$\sum_{i}^{(-)} \beta_{i} \lambda\left(\xi_{i}, r_{i}, s_{i}\right)$

where

$\alpha_{i}=\left.x_{i 0} \frac{\partial h}{\partial x_{i}}\right|_{\mathbf{x}_{0}}>0, \quad \beta_{i}=\left.x_{i 0} \frac{\partial h}{\partial x_{i}}\right|_{\mathbf{x}_{0}}<0$,

$\xi_{i}=\frac{x_{i}}{x_{i 0}}, \quad$ if $x_{i 0}>0$,

$\kappa\left(\xi_{i}, r_{i}, s_{i}\right)=\frac{\xi_{i}^{r_{i}}}{r_{i}}-\frac{\xi_{i}^{s_{i}}}{s_{i}}+\frac{1}{s_{i}}-\frac{1}{r_{i}}$,

$\lambda\left(\xi_{i}, r_{i}, s_{i}\right)=\frac{\xi_{i}^{-s_{i}}}{s_{i}}-\frac{\xi_{i}^{-r_{i}}}{r_{i}}+\frac{1}{r_{i}}-\frac{1}{s_{i}}$.

Differentiating (20) with respect to $\xi_{i}$

$\frac{\partial \kappa\left(\xi_{i}, r_{i}, s_{i}\right)}{\partial \xi_{i}}=\xi_{i}^{r_{i}-1}-\xi_{i}^{s_{i}-1}$.

The function minimum 0 can be obtained at $\xi_{i}=1$. That is

$\kappa\left(\xi_{i}, r_{i}, s_{i}\right) \geq 0$.

Similarly, it can be obtained that

$\lambda\left(\xi_{i}, r_{i}, s_{i}\right) \leq 0$.

From (23) and (24), (19) can be rewritten as

$h_{r_{i}}(\mathbf{x})-h_{s_{i}}(\mathbf{x})=\sum_{i}^{(+)} \alpha_{i} \kappa\left(\xi_{i}, r_{i}, s_{i}\right)+$

$\sum_{i}^{(-)} \beta_{i} \lambda\left(\xi_{i}, r_{i}, s_{i}\right) \geq 0$.

That is,

$h_{r_{i}}(\mathbf{x}) \geq h_{s_{i}}(\mathbf{x})$.

Therefore, the higher order approximation results in a higher approximate function value, thereby proving the conservative tendency. 